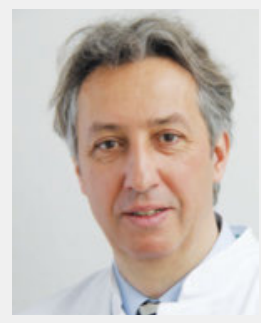

Prof. Dr. med. Stefan Thorban, München

\section{Verbesserung des Langzeit-Transplantat-Überlebens nach Nierentransplantation}

In den vergangenen 40 Jahren konnten das Ein-Jahres-Transplantat-Überleben und die Inzidenz akuter Abstoßungen im Rahmen der Nierentransplantation deutlich verbessert werden. Neben der Standardisierung chirurgischer Techniken, der Organkonservierung und dem Spendermanagement haben verbesserte immunsuppressive Strategien wesentlich zu diesen Erfolgen beigetragen, wobei die Wirkung der Immunsuppressiva aber auch kritisch gesehen werden muss. Neben signifikant hervorragenden Kurzzeitergebnissen hat sich in Bezug auf die Langzeitergebnisse hinsichtlich der Transplantatfunktion und des Transplantatüberlebens nicht viel verändert.

Die Ursachen für einen vorzeitigen Transplantatverlust sind vielfältig - angefangen von einem inzwischen akzeptierten höheren Spender- und Empfängeralter über eine höhere Komorbidität der Spender und Empfänger bis hin zu immunologischen Ursachen wie donorspezifische Antikörper, eine erhöhte Zahl an Retransplantationen und immunisierten Patienten sowie die chronische Transplantatglomerulopathie und die Spätfolgen der Immunsuppression.

Denn Calcineurininhibitoren und Steroide sind beispielsweise neben ihrer bekannten Nephrotoxizität entscheidend für die Entstehung und Verschlechterung einer Bluthochdruckerkrankung oder für die Neuentstehung von Diabetes und Hyperlipidämie verantwortlich. Die daraus resultierenden kardio- und zerebrovaskulären Erkrankungen beeinflussen die Morbidität und Mortalität der Patienten nach einer Nierentransplantation signifikant. In den vergangenen Jahren bestand der Schwerpunkt von Studien darin, mithilfe der Vermeidung von Steroiden und einer Minimierung der Calcineurininhibitoren eine individuellere, auf Langzeitfolgen abgestimmte Immunsuppression zu erreichen.

Mit der Einführung von Kostimulatorblockern, die in ersten Studien vielversprechende Ergebnisse zeigten, wurde das Interesse an einer Therapie mit Vermeidung von Calcineurininhibitoren erneut geweckt. Allerdings führte das zu frühe Absetzen von Steroiden und eine zu schnelle Reduktion oder auch Vermeidung von Calcineurininhibitoren insbesondere bei immunologischen Risikofaktoren in anderen Studien oft zu schwerwiegenden Rejektionen mit einem frühen Transplantatverlust.

Daher gilt es jetzt, Alternativen zu den derzeit vorhandenen Therapieschemata herauszuarbeiten. Die Durchführung derartiger Studien wird jedoch in Deutschland bei den derzeit bestehenden Organspendezahlen kaum zu realisieren sein. 